\title{
Insights into the antiviral functions of the RNAi machinery in penaeid shrimp
}

\author{
Yannick Labreuche ${ }^{a, *}$, Gregory W. Warr ${ }^{b, c}$
}

\begin{abstract}
a IFREMER, Département Lagons, Ecosystèmes et Aquaculture Durable en Nouvelle-Calédonie, BP 2059, 98846 Nouméa Cedex, New Caledonia, France

${ }^{\mathrm{b}}$ Division of Molecular and Cellular Biosciences, National Science Foundation, Arlington, VA 22230, USA

c Department of Biochemistry and Molecular Biology, Medical University of South Carolina, Charleston, SC 29425, USA
\end{abstract}

*: Corresponding author: Yannick Labreuche, Tel.: +33 687352588 ; fax: +33 687351177 ;

email addresses : ylabreuc@ifremer.fr ; yannick.labreuche@ifremer.fr

\begin{abstract}
:
Over the last decade, RNA interference pathways have emerged in eukaryotes as critical regulators of many diverse biological functions including, among others, transcriptional gene regulation, posttranscriptional gene silencing, heterochromatin remodelling, suppression of transposon activity, and antiviral defences. Although this gene silencing process has been reported to be relatively well conserved in species of different phyla, there are important discrepancies between plants, invertebrates and mammals. In penaeid shrimp, the existence of an intact and functional RNAi machinery is supported by a rapidly growing body of evidence. However, the extent to which this process participates to the host immune responses remains poorly defined in this non-model organism. This review summarizes our current knowledge of RNAi mechanisms in shrimp and focuses on their implication in antiviral activities and shrimp immune defences.
\end{abstract}

Keywords: Shrimp ; Innate immune response ; In vertebrate immunity ; RNA interference ; Virus 


\section{Introduction}

Shrimp are infected by more than 20 virus-caused diseases, these viruses being classified within more than 10 families that are quite diverse in their genetic material, infection cycles, geographic distribution, host range and pathogenesis (reviewed in [1, 2]). The emergence and spread of these pathogenic agents have led to considerable economic losses, threatening the economic viability and long-term sustainability of the shrimp aquaculture industry worldwide [3]. For some countries, the economic and social impacts of these pandemics have been sometimes so important that shrimp production has never fully recovered [4]. As a consequence, the need for better control and understanding of viral diseases has prompted an increasing interest in the study of the shrimp immune system, and significant advances into the molecular bases for antiviral immunity in these animals have been gained in the last years $[5,6]$.

Initially identified as a natural antiviral mechanism in plants, RNA interference (RNAi) has also been recognized as a key player in generating antiviral immunity in arthropods [7, 8]. In contrast to the considerable information available for insects for example, much less is known about how crustaceans respond to viral infections. In shrimp, gene silencing through RNAi was reported for the first time by Robalino and collaborators, who observed that in vivo administration of dsRNAs afforded in Litopenaeus vannamei protection against viral infection and thus demonstrated that marine invertebrates were able to display inducible antiviral immunity in response to a virus-associated molecular structure $[9,10]$. Following these observations, RNAi-based applications (to down-regulate both endogenous shrimp genes and viral genes) have generated substantial enthusiasm and enabled a wide field of research in shrimp, as reflected by the increasing number of publications on this topic. Becoming a standard laboratory practice, RNAi technology has been increasingly used to unravel gene functions in vivo, providing new opportunities not only to understand in greater depth the nature of shrimp biological and physiological processes, but also to decipher host-pathogen interactions. Based on these approaches, the delivery of dsRNAs or siRNAs specific for viral genes into shrimp has proved feasible and highly effective to suppress viral replication of the most intensively studied viruses, namely the white spot syndrome virus (WSSV), the yellow head virus (YHV) and the Taura syndrome virus (TSV). The practical aspects of developing RNAi-based therapies for viral diseases (i.e. choice of RNAi molecules, delivery routes/methods, targeted genes, etc) have been extensively covered elsewhere [11-14] and are outside the scope of this review. Instead, we present in this article an overview of the current knowledge of the RNAi machinery in penaeid shrimp and discuss the implication of RNAi components in the shrimp response to viral infections

\section{RNAi silencing pathways : a brief overview}

First described in plants and nematodes [15], and subsequently in many eukaryotes, RNAi is a highly conserved nucleic acid-based mechanism, playing roles both in the regulation of endogenous gene expression as well as in antiviral defence mechanisms in plants, animals and fungi. The basic RNAi process is frequently divided into three major steps [16]. First, the engagement of this machinery is initiated by long double-stranded RNAs (dsRNAs) produced locally or taken up by the cells. These precursors are processed by a member of the Dicer family of RNase-III-like enzymes into small effector RNA duplexes (sRNAs, 20-30 nt), among which microRNAs (miRNAs) and small interfering RNAs (siRNAs) represent the two major classes. siRNAs originate from endogenous transcripts from repetitive sequences, exogenous dsRNAs (e.g., viral RNAs) or from transcripts that can form long hairpins, while miRNAs are derived from endogenous, single-stranded noncoding RNA transcripts or introns that fold into imperfect stem-loop structures. In a second step, these duplexes are unwound 
and one single-stranded RNA, called the guide strand, is preferentially loaded into a multimeric protein complex, the RNA-induced silencing complex (RISC). Third, this complex, of which an Argonaute (Ago) family protein forms the catalytic core, searches the transcriptome and, depending on the sequence homology between the potential target messenger RNAs (mRNAs) and the guide strand, induces post-transcriptional gene silencing via a variety of mechanisms. While siRNAs generally fully match their mRNA targets, miRNAs can target mRNAs that are not fully complementary, inducing mRNA degradation and/or translational repression.

\section{A functional RNAi pathway in shrimp}

Although RNAi pathways comprise different proteins and mechanisms in species of different phyla, core RNAi components have been described in the major branches of eukaryotes, indicating that these pathways may share both evolutionarily conserved and convergent mechanisms. dsRNA-induced gene silencing has been reported in several shrimp species, suggesting the existence of an intact RNAi machinery. Because the understanding of the molecular mechanisms underlying RNAi in shrimp may help in applying RNAi-derived techniques to these organisms, research efforts have focussed in the last decade on the identification and functional characterization of RNAi pathway components (amongst which are Dicer and Argonaute) in penaeids (Table 1). Dicer proteins are a widely conserved family, present in many organisms including plants, fungi, and the Metazoa. Depending on the organism, there may be one or more Dicer-like enzymes. The plants Arabidopsis thaliana and Oryza sativa contain four and five Dicer proteins, respectively, while metazoans contain only one (Caenorhabditis elegans and vertebrates), two (insects and cnidarians) or five (poriferans and placozoans) Dicer genes [17-19]. To date, only one Dicer gene has been identified in Penaeus monodon and Marsupenaeus japonicus, (PmDcr1 and MjDcr1, respectively) while two Dicer-like genes ( $L v D c r 1$ and $L v D c r 2)$, have been cloned in L. vannamei [20-22]. In plants and insects, each Dicer protein is involved in the biogenesis of different types of small-silencing RNAs with distinct characteristics [23, 24]. In insects, Dicer1 is mainly used to produce miRNAs, while Dicer-2 participates in the processing of long dsRNAs (including those from viral origin) into siRNAs [17] (Figure 1). Structural and phylogenetic analyses of the shrimp Dicer family have established that $L v D c r 1, P m D c r 1$ and MjDcr1 encode putative proteins containing between 2473 and 2482 amino acids (aa), sharing more than 95\% identity and clustering with the Dicer-1 group from other invertebrates and vertebrates, while the putative protein encoded by LvDcr2 is 1502 aa in size and shares only $30 \%$ identity with shrimp Dcr1s, grouping with the Dicer-2 proteins characterized in insects and cnidarians. Although mRNA transcripts of shrimp Dcrs have been detected in several shrimp tissues [20,22], it still remains to be determined whether these proteins possess specialized functional activities in the processing of small effector RNA duplexes.

To recruit Argonaute proteins and form the core of the RNA-induced silencing complex, Dicer family enzymes have been shown to act in cooperation with several dsRNA binding proteins, including the HIV-1 transactivating response (TAR) RNA-binding protein (TRBP) and a protein activator of PKR (PACT) in humans [25-27] or R2D2 and Loquacious (LOQS) in Drosophila melanogaster [28, 29] (Figure 1). Other accessory proteins including the eukaryotic initiation factor 6 (elF6) have also been described as components of this multiprotein complex [30]. Several new members of the TRBP family have been characterized in Fenneropenaeus chinensis (Fc-TRBP1-3) and M. japonicus (Mj-TRBP1-3) and shown to share more than $99 \%$ sequence similarity [31, 32] (Table 1). A TRBP homologue was also recently isolated in L. vannamei [21]. Phylogenetic analysis and sequence alignments from multiple species demonstrated that these shrimp TRBPs are highly conserved in relationship to other members of the same family, especially in terms of 
dsRNA-binding domains (dsRBDs). Homologues of elF6 (namely $F_{c-\text {-elF6 }}$ and Mj-elF6) were also cloned in $F$. chinensis and $M$. japonicus and reported to be highly similar to the elF6 sequences in other species [31, 32]. Similar to reports in other organisms [30], direct interactions were evidenced between elF6 and TRBP in shrimp, suggesting their association in the same protein complex. Moreover, knockdown of shrimp TRBP and elF6 was shown to result in a substantial loss of dsRNA-mediated silencing of other target mRNAs, which is consistent with the hypothesis that these two proteins function as part of the shrimp RNAi machinery [31, 32].

Argonaute protein family members, the key components of the RISC, were the first components of a putative RNAi pathway to be identified in penaeid shrimp [33]. As is the case with the Dicer family, there may be one or more Argonaute family members within a given organism. For instance, the yeast Schizosaccharomyces pombe contains only one Argonaute, while five paralogues have been found in Drosophila, eight in humans, ten in Arabidopsis and twenty-seven in C. elegans [34] (Table 1). These Argonaute proteins can be divided into two subclasses, namely the AGO and PIWI subfamilies. Members of the Ago subfamily are ubiquitously expressed and associate with miRNAs and siRNAs, whereas the expression pattern of PIWI subfamily members is mainly restricted to the germline, explaining why this subfamily is considered to play a tissue-specific role during development rather than a more ubiquitous role in RNAi [34]. In the black tiger shrimp P. monodon, two isoforms (Pm-Ago and Pem-AGO) were cloned [33, 35], while L. vannamei was reported to contain two different Argonaute proteins (Lv-Ago 1 and Lv-Ago 2) [36]. The corresponding genes were found to be ubiquitously expressed and their deduced amino acid sequences shown to possess the two distinctive features of the AGO subfamily, i.e. a PAZ domain involved in dsRNA binding and a PIWI domain reported to display RNase activity, suggesting thus their implication in RNAi mechanisms [33, 35, 36]. A confirmation of this hypothesis was provided by the knock-down of Pem-AGO in P. monodon, that was reported to partially reduce $(\approx 50 \%$ of the original activity) the efficiency of dsRNA-mediated silencing of an endogenous shrimp gene [35]. Considering these results, it has been hypothesized that other Argonaute family members present in shrimp may be involved in this mechanism. Supporting this assumption, the study of $L v$-ago 1 and $L v$-ago 2 transcript accumulation following dsRNA injection into shrimp revealed a strong induction of $L v$-ago 2 mRNA expression only [36], while co-immunoprecipitation and pull-down assays demonstrated that Lv-Ago 2 interacts with LvDcr2 and LvTRBP1 [21]. Taken together, these data suggest that the two Argonaute proteins characterized in L. vannamei may play specialized functions, Lv-Ago 2 being more specifically implicated in a "siRNAi pathway". Similar observations have been previously reported in Drosophila, where Ago1 and Ago2-RISC are functionally distinct and allow the silencing of different types of target RNAs by distinct mechanisms. miRNAs partition thus between Ago1- and Ago2-RISC, while siRNAs associate almost exclusively with Ago2-RISC [37] (Figure 1). Whether similar mechanisms of Argonaute specialization occur in shrimp remains to be formally demonstrated.

Many other RISC-associated proteins involved in posttranscriptional regulation have been characterized in vertebrate and invertebrate animal models, among which is the Tudor Staphylococcal nuclease (TSN) [38]. A TSN homologue (PmTSN) was recently characterized in P. monodon [39]. Because knockdown of PmTSN only partially reduced the efficiency of dsRNA-mediated gene silencing, it has been proposed that this protein may function as a minor component affecting RISC efficiency but dispensable for RNAi in shrimp.

As indicated by the different studies presented here, considerable advances have been made in the characterization of a RNAi machinery in penaeids. However there are still many open questions to be solved. For instance, the discovery of different sets of Dicer and Argonaute proteins in $L$. vannamei suggest the potential existence of several RNAi pathways in shrimp. Whether these pathways are redundant or whether they have specialized independent functions has yet to be determined. In vivo investigations of gene functions at 
the organism level are thus clearly needed. It is well known that transgenic and knockout animal technology are not available or are unreliable in shrimp, but it is possible, as exemplified by different research articles cited in this review [32, 35], to silence the silencing machinery itself by injection of long dsRNA for a given target (a process also known as "RNAi of RNAi") . However, silencing of some genes presumptively associated with the RNAi pathway has been sometimes shown to result in a lethal phenotype, as observed by Labreuche and collaborators in L. vannamei [36], suggesting that these components may be involved in functions essential for life but which remain largely uncharacterized. To begin addressing this issue, we next focus in this review on the role of the RNAi machinery in shrimp antiviral immunity.

\section{RNAi as an antiviral mechanism in penaeids ?}

Mammals are frequently considered to have lost antiviral silencing activities and their innate antiviral defences thus rely mainly on the interferon response [40]. In contrast, RNAi has evolved as a potent antiviral pathway in plants and invertebrates, presumably as a means of responding to viral infections through the sensing of dsRNA, a central molecular pattern found in viral genomes, in viral replication complexes or resulting from bidirectional transcription in DNA viruses. As mentioned above, two Rnase III-related Dicers initiate the miRNA and siRNA pathways in D. melanogaster (DCR1 and DCR2, respectively). However, recognition of viral dsRNA relies essentially and specifically on DCR2, leading to the production of virus-derived siRNAs (also designated vsiRNAs). These vsiRNAs are transferred with the help of R2D2 (a dsRNA binding protein) to AGO2, which targets the viral RNA molecules for degradation [41, 42]. Unlike D. melanogaster, a functional redundancy among the Dicer-like proteins (DCLs) has been reported in A. thaliana, DCL4 and DCL2 producing vsiRNAs against distinct positive-strand RNA viruses in a hierarchical and redundant manner $[43,44]$. To decipher the functional role(s) of Dicer and Argonaute proteins in shrimp antiviral immunity, several studies have first attempted to investigate the mRNA transcript abundances of the corresponding genes following experimental viral challenges [20-22]. The underlying idea was that if these components are effector mechanisms of the shrimp antiviral defences, their expression would be modified during the course of infection. The expression of PmDcr1 was thus investigated and shown to be transiently up-regulated at an early stage of gill-associated virus (GAV) infection ( $<48 \mathrm{~h}$ ), but returned to normal levels later and no direct correlation could be found between levels of PmDcr1 expression and the viral loads in shrimp [22]. The expression pattern of LvDcr1 in hemocytes and gills of shrimp challenged with TSV was somewhat similar, being upregulated in both tissues during the first $24 \mathrm{~h}$ after TSV injection and subsequently decreasing [20]. In contrast to these results, no significant difference occurred at $24 \mathrm{~h}$ postchallenge in the expression level of LvDcr1 mRNA between infected animals and the PBSinjected controls following WSSV challenge [45]; however, the temporal mRNA expression of LvDcr2 increased continuously during the first $48 \mathrm{~h}$ after WSSV challenge [21]. Finally, the expression of Pm Ago mRNA was reported to be 2-3-fold increased during the early period of viral infection but thereafter declined rapidly. Interestingly, experimental infection by a bacterial pathogen did not modify Pm Ago transcript abundance, leading the authors to propose that its expression was specifically associated with virus infection [33]. Although the expression patterns of the shrimp Dicer and Argonaute genes in viral response studies provide the first clues for the antiviral role of their cognate protein, it is quite difficult to draw any definitive conclusion as to whether or not these proteins play a direct role in shrimp defences. Moreover, the different outcomes observed in LvDcr1 gene expression after viral infection [20,45] could be due to a lack of standardized challenge protocols and bioassays, to the existence of genetic variations in the viral stocks used, and/or to the use of domesticated stocks of shrimp genetically selected for tolerance to some viruses (see [46] for a review). More direct evidence has come from PmDcr1 knockdown by injection of 
sequence-specific dsRNA prior to infection with low viral titres [22]. In this experimental system, higher viral loads and more rapid mortalities occurred in PmDcr1-silenced shrimp, illustrating a significant enhancement of the animal's susceptibility to viral infection. Although the $L v D c r 2$ expression pattern differed from that of $L v D c r 1$ in $L$. vannamei, both were clearly induced following viral challenge, suggesting that shrimp Dcr1 and Dcr2 may both participate in the antiviral immune response. Whether there is a certain form of functional redundancy among these Dicer proteins in shrimp (as observed in plants), or whether LvDcr1 and LvDcr2 act by distinct mechanisms, still remains to be elucidated by knockdown experiments for example. In addition, more compelling evidence is needed to better decipher the implication of shrimp Argonautes in the RNAi-mediated inhibition of viral replication. Other putative shrimp RNAi components have been investigated for their role in antiviral RNAi. Complex changes in the expression patterns of TRBP and elF6 were thus detected in F. chinensis in response to WSSV challenge [31]. However, the most straightforward demonstration of the implication of TRBP in antiviral immunity came from co-injection experiments of recombinantly expressed $F_{C}$-TRBP1 with WSSV into shrimp, that were shown to induce an inhibition of viral replication. These results were recently confirmed in $M$. japonicus by dsRNA-mediated silencing of Mj-TRBP and Mj-elF6 which was reported to increase WSSV replication, implying a crucial role of these proteins in the antiviral defence program [32].

In invertebrates, much of the understanding of antiviral RNAi derives from studies on $D$. melanogaster and $C$. elegans, two genetically tractable animal models where disruption of gene function is a classic approach in genetic analyses. In the absence of similar technology in understudied animals such as shrimp, our knowledge about the implication of the RNAi machinery in antiviral immunity remains much more scarce in comparison. However, different studies have provided several lines of evidence clearly supporting a naturally antiviral role for RNA silencing in shrimp. Research in this field is still in its infancy and needs to be further pursued to help build a global picture of the pathway(s) controlling viral infections and transmission.

\section{Antiviral cellular miRNAs in shrimp?}

miRNAs are generated from miRNA-encoding genes and are 21- to 23-nucleotide ssRNAs considered to be key regulators of the expression of genes with roles in many biological processes including development, cellular differentiation, proliferation, metabolism and apoptosis [47]. They thus represent an additional level of gene expression regulation between mRNA and protein translation (see [48, 49] for a review). They are transcribed as long, hairpin-containing precursors (referred to as primary miRNAs or pri-miRNAs) by RNA polymerase II and cleaved in the nucleus by the Microprocessor, a protein complex which includes the RNase III enzyme Drosha, and its binding partner known as Pasha in D. melanogaster or DGCR8 in humans (Figure 1). Recently, it has been demonstrated that other proteins, including Ars2 (Arsenite-resistance protein 2), physically interacts with the Microprocessor [50]. Next, the pre-miRNAs are transported to the cytoplasm, where they are further processed by a member of the Dicer family (DCL-1 in Arabidopsis and DCR1 in Drosophila) and its cofactor TRBP (or LOQS in Drosophila) into mature miRNAs, and finally loaded into a RISC. Because recognition by the miRISC of a target mRNA relies on the complementary base-pairing of a 6-8 nt seed region, a single miRNA can mediate translational inhibition of hundreds of proteins at once. Since the discovery of this pathway, more than 18000 miRNAs have been annotated, according to the last miRBase database release in November 2011 (http://www.mirbase.org/), in plants and animals, among which are a few aquatic crustacean species [51,52].

In L. vannamei, the identification of two Argonaute and two Dicer paralogues, combined with the fact that only $L v$-ago2 expression was responsive to dsRNAs while the substrate for LvAgo1 remained enigmatic, has led to the suggestion that a miRNA pathway may also be 
operating in penaeid shrimp [36]. This hypothesis was indirectly reinforced by the report that long-hairpin mRNAs were also able to promote targeted gene silencing in $P$. monodon [53]. Finally, the first evidence has come last year from a small RNA cloning approach in the kuruma shrimp $M$. japonicus, that has allowed for the first time the identification in shrimp of 35 miRNAs of which 15 were shown to display high homologies with known miRNAs from other Metazoa [54]. Following this discovery, homologues of two proteins involved in miRNA biogenesis (namely LvArs2 and LvPasha) have been characterized in L. vannamei, their deduced amino acid sequence sharing in both cases more than $40 \%$ identity with their insect orthologues [55]. In D. melanogaster, Ars2 plays a role in several modes of RNA silencing, regulating the siRNA pathway by associating with DCR2 and modulating its activity in vitro, but also controlling the miRNA pathway by interacting with Pasha and stabilizing pri-miRNAs [50]. Similarly to the observations reported in this insect model, it was demonstrated by means of co-immunoprecipitation assays that the shrimp LvArs2 interacts with LvPasha and LvDcr2 [55]. Although still largely unclear, the emerging picture suggests that, as in other arthropods [56], multiple small RNA regulatory pathways function in shrimp.

In a broad range of eukaryotic species, much of the understanding of the cellular processes modulated by the miRNA pathway has focused on development. However, a role for miRNAs in the antiviral defense network has gradually been proposed $[57,58]$. In $M$. japonicus, the expression level of the 35 identified miRNAs was examined upon WSSV infection. Thirteen were reported to be upregulated, and 15 downregulated after viral challenge, leading the authors to propose their putative implication in the shrimp immune response [54]. Several publications have reported in other organisms similar modifications in the host miRNA expression profile following viral infection [59-61]. The problem with such approaches is the difficulty of determining if the observed changes are elicited by the pathogen itself to enhance its infectious cycle, or instead if they occur to prevent viral replication, participating thus in the host immune responses. Indeed, it is well known that viruses have evolved multiple mechanisms to interfere with host cell regulation and render the infected cells amenable to viral replication, producing thus foreign molecules that may trigger systemic warnings and responses [62]. As stated by the authors of these studies, additional experiments have thus to be conducted to explore the individual role(s) of these shrimp miRNAs. Following the approach developed to investigate the implication of candidate genes in the RNAi antiviral response, the expression patterns of LvArs2 and LvPasha were also examined in shrimp stimulated by the injection of poly(C-G), a commercial dsRNA analogue [55]. Although the mRNA transcript abundance of both genes was modified following the immune stimulation, the results are insufficient to answer the question of the implication of these components in the shrimp immune response. It is worth reiterating that functional studies based on reverse genetic approaches will be required to fully understand the mechanistic role of these proteins in shrimp physiology and immune defence.

\section{6. dsRNA-induced antiviral immunity in penaeids}

As mentioned earlier, long dsRNAs are recognized by the host cells as a pathogenassociated molecular pattern (PAMP). In vertebrates, dsRNA sensing rapidly initiates host immune defenses via complex intracellular signaling pathways which culminate in the induction of inflammatory responses mediated by various transcription factors, including IFN regulatory factors (IRFs) and nuclear factor (NF)-KB [63-65]. Similar innate responses to dsRNA were assumed for a long time to have been absent from the arthropod immune system (including that of insects, shrimp and other crustaceans), mainly because of the lack of invertebrate homologues of IFNs and IFN receptors. However, while using dsRNAmediated gene silencing to investigate the role of specific host genes in shrimp immune response, Robalino and collaborators fortuitously observed that administration of dsRNA of arbitrary sequence also elicited in L. vannamei a partial protection from viral infection [9]. 
Similar results were subsequently reported in different shrimp species by several authors (see $[13,66,67]$ for reviews), suggesting the existence of a possible evolutionary link between innate antiviral immunity in vertebrates and invertebrates. The recent characterization of an interferon-like protein in $P$. japonicus [68] and the cloning of a $Y$ interferon-inducible lysosomal thiol reductase homologue (an enzyme shown to be induced by interferon- $\gamma$ ) in P. monodon [69], together with the observed improved survival following "vaccination" [70, 71], further support the idea that the traditional views of innate immune responses in shrimp may need to be reconsidered.

To date, the mechanisms underlying the dsRNA-induced antiviral immunity in shrimp remain largely unknown. In vertebrates, the general non-specific antiviral response was initially considered as being initiated by long dsRNAs only, and shorther RNA duplexes (such as siRNAs) were therefore used for specific gene manipulation in order to circumvent these side effects [72]. However, it was later reported that siRNAs could also activate cells of the immune system and induce the production of cytokines [73]. In L. vannamei, the non-specific effect of arbitrary dsRNAs was reported to be length-dependent since only $>50$ bp-dsRNAs, but not siRNAs, could generate antiviral protection during a viral challenge $[10,36]$. Nevertheless, discrepancies in the ability of siRNAs to promote this side effect have also been reported in shrimp [74]. In vertebrates, the extent to which the innate immune response may be activated is known to be dependent on the siRNA sequence but also on the type of delivery system employed for cell transfection [75, 76]. It remains to be explored in shrimp whether similar considerations may applied to explain the conflicting results sometimes observed between studies.

To further understand the mechanistic details of the innate response to dsRNA in penaeids, several approaches have been explored, among which is the identification of the receptor(s) involved in dsRNA recognition. In vertebrates, antiviral responses are well-characterized and initiated through the detection of viral nucleic acids by pattern recognition receptors (PRRs) that belong to two families, the retinoic acid-inducible gene I (RIG-I)-like receptors (RLRs) and the transmembrane Toll-like receptors (TLRs), detecting viral components outside of cells as well as in cytoplasmic vacuoles after phagocytosis or endocytosis (see [77] for a review). Among the 10 TLRs identified in humans, only 4 are involved in the detection of nucleic acids derived from viruses and bacteria: TLR7 and TLR8 recognize sRNAs, TLR9 ssDNAs, while TLR3 senses dsRNAs independently of its base sequence [78]. Searches of shrimp sequences have not yet yielded homologues for RLRs, whereas a set of TLRs have been identified in L. vannamei [79, 80], F. chinensis [81], P. monodon [82] and M. japonicus [83]. It was hypothesized that these shrimp TLRs may contribute to dsRNA sensing, and the role of LvToll (later renamed LvToll1 following the characterization in L. vannamei of two novel Toll-like receptors, LvToll2 and LvToll3), was thus investigated by a reverse genetic approach [84]. However, the results were inconclusive. To date, most of the information about shrimp TLR functions comes mainly from correlative expression analyses. Although recent work has shown that relative expression of LvToll1, LvToll2 and LvToll3 was upregulated after viral challenge [80], it is still far from clear whether one of these receptors may act as a dsRNA sensor activating specific signaling pathway(s).

In vertebrates, once PRRs detect dsRNAs, a chain of events is activated to promote inhibition of protein synthesis, transcriptional induction of interferon and other cytokines, and ultimately, cell death. Several distinct pathways responsible for each of these events have been described and reported to display some degree of crosstalk between them [85]. For instance, the RNA-activated protein kinase (PKR), one of the most studied components of the IFN-induced antiviral pathways, is activated by dsRNAs and exerts its antiviral functions by translational regulation [86]. Interestingly, its activity has been shown to be modulated by multiple viral and cellular factors, among which are TRBP and PACT, two components of the RNA-induced silencing complex (RISC) [87]. PACT association with PKR was thus reported to activate PKR in the absence of dsRNAs [88], whereas interaction of TRBP with PKR leads 
to a strong inhibition of the kinase by direct binding [89]. In shrimp, Robalino and collaborators have proposed the concept that the mechanisms responsible for dsRNAinduced innate immunity and RNAi in the antiviral response may crosstalk and interact functionally, facilitating the host antiviral defence $[10,66]$. Supporting this hypothesis, it was later shown that non-specific dsRNAs trigger induction of $L v$-ago 2 mRNA expression in L. vannamei, suggesting the existence of potential functional overlap or link(s) between nonspecific activation of antiviral immunity and the RNAi mechanism [36]. Considering the recent characterization of several shrimp TRBPs [21, 31, 32], it would be interesting to investigate whether these components may also be involved in general antiviral immunity as described in mammals. Indeed, as previously mentioned, dsRNA-mediated silencing of Mj-TRBP was clearly shown to promote viral replication [32]. It remains to be precisely determined if this increased number of WSSV DNA copies results solely from an impairment of the RNAi machinery through a potential destabilization of Dicer following TRBP depletion or may, to some extent, be an indirect consequence from a decreased efficiency of the non-specific antiviral immune response due to a potential dual role played by this protein.

Although a sequence-independent antiviral response has been described in insects, such as the sandfly Lutzomyia longipalpis [90] or the silkworm Bombyx mori [91], there are no similar reports to date in commonly studied model organisms with fully sequenced genomes, such as Drosophila. This may in part explain why the question of the mechanisms underpinning this phenomenon has raised relatively limited interest in the scientific community. The development of functional genomic and proteomic approaches in penaeid shrimp (see [5] for a review) should help obtaining new molecular information and thus fill the knowledge gap between marine invertebrates and other well-developed biological models.

\section{Interplay between viral infections and the RNAi machinery}

To counteract host defences, replicate in host cells and thus achieve systemic infection, viruses have evolved several original strategies that frequently rely on the subversion of the host immune signalling pathways for their own benefit. Among these strategies is the production of viral suppressors of RNAi (also known as VSRs) to inhibit various stages of the silencing process. This phenomenon was first described in plant viruses [92], but many other plant as well as animal viruses displaying the same ability have rapidly been identified (reviewed in [93, 94]). Encoded by ORFs within or overlapping with other conserved viral genes, the VSRs characterized so far are all dsRNA-binding proteins that greatly differ not only in their primary sequence and structure but also in their function, targeting different key steps of the RNAi pathway. Some VSRs have thus been reported to suppress siRNA production, while others act by either sequestering siRNAs to prevent their incorporation into the RISC or preventing short- and long-distance spread of the RNA silencing effect. Finally, VSRs have also been proposed to alter the function of host miRNAs (reviewed in $[93,95]$ ).

Given the suspected importance of RNAi as an antiviral defence mechanism in shrimp, it may be hypothesized that shrimp viruses may similarly encode VSRs to counteract the host antiviral responses. This assumption was verified quite recently in L. vannamei when Robalino and collaborators reported for the first time the inhibition of dsRNA-mediated silencing in WSSV-infected shrimp [66]. Interestingly, this inhibitory effect was shown to take place preferentially in certain tissues such as the hepatopancreas, an organ considered as a primary site for the production of immune response factors [96]. Although it occurred more occasionally, a similar suppression of gene silencing was also observed by the authors in the hepatopancreas of TSV-infected shrimp. To date, the exact mechanisms supporting the VSR activities of these two viruses remain to be determined. Moreover, additional experiments are needed to investigate whether other shrimp viruses may display the same ability. For instance, the Mourilyan virus (MoV), that infects $P$. monodon and M. japonicus shrimp in 
Australia, comprises 4 RNA segments, one of which encodes a putative small non structural protein (NSs2) [97]. Because bioinformatic analyses showed that NSs2 is similar in size to the NSs proteins of several arthropod-borne bunyaviruses known to display RNAi suppression activity, a similar function was suspected in shrimp. However, knockdown of MoV NSs2 expression did not reduce the viral loads nor modify targeted silencing of other genes [98]. Further studies are thus needed to decipher the NSs2 function in MoV pathogenesis. Criteria to predict RNA silencing suppressor activity in viral proteins have been recently provided based on the characteristics possessed by VSRs of plant and animals viruses [93, 99]. They include the presence of dsRNA-binding domains as well as the detection of glycine-tryptophane/tryptophane-glycine repeats (GW/WG) within the protein sequence. In depth analyses of available sequenced genomes from shrimp viruses may thus represent a first step toward the identification of potential VSR activities in shrimp viruses.

\section{Concluding remarks}

Although the lack of whole genome sequences in penaeid shrimp represents a serious impediment to the understanding of the molecular immune responses, an increasing number of sequence information is now available in public databases, allowing in the past few years the identification of several core components of the machinery for RNA-mediated silencing in shrimp. However, our knowledge of this biological system is still far from complete and many important questions remain to be addressed in the future: how many types of RNAi pathways are there exactly in shrimp ? What are their roles? How these pathways are regulated ? Do they cooperate ? The rapid development of functional genomics and proteomics in shrimp should undoubtedly help to discover new players of silencing pathway(s) and decipher their biological implication(s).

One other major challenge in shrimp will be to understand how these RNAi components participate in the fine-tuning of innate immune signaling pathways. Despite the evidence presented here for the important role of RNAi in antiviral defences, additional experiments supporting this scenario are needed. One of the landmarks of the antiviral RNAi response is the production of virus-derived siRNAs from viral dsRNAs. It would be particularly interesting to determine whether infected animal's tissues contain significant amounts of vsiRNAs, since this would indicate that viruses are both inducers and targets of the shrimp antiviral silencing machinery.

Finally, it is clear that shrimp immune responses, like all complex cellular phenomena, will need to be understood not just in terms of the properties of the individual molecular components involved, but ultimately in terms of the interactions of those components in the integrated functional networks that determine the behavior of cells. Such approaches are in their infancy in shrimp [100-102] but should ultimately provide a basis for generating predictive hypotheses to guide future research in crustacean host-virus interactions.

\section{Acknowledgements}

The authors warmly thank Javier Robalino for helpful comments and critical reading of the manuscript. Y.L is supported by the DEDUCTION project funded by the Institut Français de Recherche et d'Exploitation de la Mer (IFREMER), the Provincial Institutions (Province Sud, Province Nord et Province des lles Loyauté) and by the Government of New Caledonia. Any opinions, findings and conclusions or recommendations expressed in this material are those of the authors and do not necessarily reflect the views of the supporting bodies mentioned herein. 


\section{References}

1. Bonami J-R. Shrimp Viruses. Encyclopedia of Virology, Third Edition (BWJ Mahy and MHV Van Regenmortel, Editors) Oxford: Elsevier. 2008 4:567-76.

2. Lightner DV. Virus diseases of farmed shrimp in the Western Hemisphere (the Americas): A review. J Invertebr Pathol. 2011 106:110-30.

3. Walker PJ, Mohan CV. Viral disease emergence in shrimp aquaculture: origins, impact and the effectiveness of health management strategies. Rev Aquac. 2009 1:125-54.

4. Walker PJ, Winton JR. Emerging viral diseases of fish and shrimp. Vet Res. 2010 41:51.

5. Robalino J, Carnegie RB, O'Leary N, Ouvry-Patat SA, de la Vega E, Prior S, et al. Contributions of functional genomics and proteomics to the study of immune responses in the Pacific white leg shrimp Litopenaeus vannamei. Vet Immunol Immunopathol 2009 128:110-8.

6. Liu H, Söderhäll K, Jiravanichpaisal P. Antiviral immunity in crustaceans. Fish Shellfish Immunol. 2009 27:79-88.

7. Wang XH, Aliyari R, Li WX, Li HW, Kim K, Carthew R, et al. RNA interference directs innate immunity against viruses in adult Drosophila. Science. 2006 312:452-4.

8. Zambon RA, Vakharia VN, Wu LP. RNAi is an antiviral immune response against a dsRNA virus in Drosophila melanogaster. Cell Microbiol. 2006 8:880-9.

9. Robalino J, Browdy CL, Prior S, Metz A, Parnell P, Gross P, et al. Induction of Antiviral Immunity by Double-Stranded RNA in a Marine Invertebrate. J Virol 2004 78:10442-8.

10. Robalino J, Bartlett T, Shepard E, Prior S, Jaramillo G, Scura E, et al. Double-stranded RNA induces sequence-specific antiviral silencing in addition to nonspecific immunity in a marine shrimp: convergence of RNA interference and innate immunity in the invertebrate antiviral response? J Virol. 2005 79:13561-71.

11. Krishnan P, Gireesh-Babu P, Rajendran KV, Chaudhari A. RNA interference-based therapeutics for shrimp viral diseases. Dis Aquat Organ. 2009 86:263-72.

12. Escobedo-Bonilla CM. Application of RNA Interference (RNAi) against Viral Infections in Shrimp: A Review. J Antivir Antiretrovir. 2011 S9:doi:10.4172/jaa.S9-001.

13. Hirono I, Fagutao FF, Kondo H, Aoki T. Uncovering the mechanisms of shrimp innate immune response by RNA interference. Mar Biotechnol (NY). 2011 13:622-8.

14. Robalino J, Chapman RW, de la Vega E, O'Leary NA, Gorbach DM, Du Z-Q, et al. Advances in Genomics and Genetics of Penaeid Shrimp. In: Fletcher GL, Rise ML, editors. Aquaculture Biotechnology: Wiley-Blackwell, Oxford, UK; 2011, p. 21-42.

15. Fire A, Xu S, Montgomery MK, Kostas SA, Driver SE, Mello CC. Potent and specific genetic interference by double-stranded RNA in Caenorhabditis elegans. Nature. 1998 391:806-11.

16. Siomi H, Siomi MC. On the road to reading the RNA-interference code. Nature. 2009 457:396-404. 
17. Lee YS, Nakahara K, Pham JW, Kim K, He Z, Sontheimer EJ, et al. Distinct roles for Drosophila Dicer-1 and Dicer-2 in the siRNA/miRNA silencing pathways. Cell. 2004 117:6981.

18. de Jong D, Eitel M, Jakob W, Osigus H-J, Hadrys $H$, DeSalle R, et al. Multiple Dicer Genes in the Early-Diverging Metazoa. Mol Biol Evol. 2009 26:1333-40.

19. Ketting RF, Fischer SE, Bernstein E, Sijen T, Hannon GJ, Plasterk RH. Dicer functions in RNA interference and in synthesis of small RNA involved in developmental timing in $C$. elegans. Genes Dev. 2001 15:2654-9.

20. Yao X, Wang L, Song L, Zhang H, Dong C, Zhang Y, et al. A Dicer-1 gene from white shrimp Litopenaeus vannamei: Expression pattern in the processes of immune response and larval development. Fish Shellfish Immunol. 2010 29:565-70.

21. Chen $Y-H$, Jia X-T, Zhao L, Li C-Z, Zhang S, Chen Y-G, et al. Identification and functional characterization of Dicer2 and five single VWC domain proteins of Litopenaeus vannamei. Dev Comp Immunol. 2011 35:661-71.

22. Su J, Oanh DTH, Lyons RE, Leeton L, van Hulten MCW, Tan S-H, et al. A key gene of the RNA interference pathway in the black tiger shrimp, Penaeus monodon: Identification and functional characterisation of Dicer-1. Fish Shellfish Immunol. 2008 24:223-33.

23. Li L, Liu Y. Diverse small non-coding RNAs in RNA interference pathways. Methods Mol Biol. 2011 764:169-82.

24. Melnyk CW, Molnar A, Baulcombe DC. Intercellular and systemic movement of RNA silencing signals. EMBO J. 2011 30:3553-63.

25. Chendrimada TP, Gregory RI, Kumaraswamy E, Norman J, Cooch N, Nishikura K, et al. TRBP recruits the Dicer complex to Ago2 for microRNA processing and gene silencing. Nature. 2005 436:740-4.

26. Haase AD, Jaskiewicz L, Zhang $H$, Laine $S$, Sack R, Gatignol A, et al. TRBP, a regulator of cellular PKR and HIV-1 virus expression, interacts with Dicer and functions in RNA silencing. EMBO Rep. 2005 6:961-7.

27. Kok $\mathrm{KH}, \mathrm{Ng} \mathrm{MH}$, Ching YP, Jin DY. Human TRBP and PACT directly interact with each other and associate with dicer to facilitate the production of small interfering RNA. J Biol Chem. 2007 282:17649-57.

28. Okamura K, Robine N, Liu Y, Liu Q, Lai EC. R2D2 organizes small regulatory RNA pathways in Drosophila. Mol Cell Biol. 2011 31:884-96.

29. Forstemann K, Tomari Y, Du T, Vagin VV, Denli AM, Bratu DP, et al. Normal microRNA maturation and germ-line stem cell maintenance requires Loquacious, a double-stranded RNA-binding domain protein. PLoS Biol. 20053.

30. Chendrimada TP, Finn KJ, Ji X, Baillat D, Gregory RI, Liebhaber SA, et al. MicroRNA silencing through RISC recruitment of elF6. Nature. 2007 447:823-8.

31. Wang S, Liu N, Chen A-J, Zhao X-F, Wang J-X. TRBP Homolog Interacts with Eukaryotic Initiation Factor 6 (elF6) in Fenneropenaeus chinensis. J Immunol. 2009 182:5250-8. 
32. Wang S, Chen AJ, Shi LJ, Zhao XF, Wang JX. TRBP and elF6 Homologue in Marsupenaeus japonicus Play Crucial Roles in Antiviral Response. PLoS One. 2012 7:e30057.

33. Unajak S, Boonsaeng V, Jitrapakdee S. Isolation and characterization of cDNA encoding Argonaute, a component of RNA silencing in shrimp (Penaeus monodon). Comp Biochem Physiol B Biochem Mol Biol 2006 145:179-87.

34. Tolia NH, Joshua-Tor L. Slicer and the argonautes. Nat Chem Biol. 2007 3:36-43.

35. Dechklar M, Udomkit A, Panyim S. Characterization of Argonaute cDNA from Penaeus monodon and implication of its role in RNA interference. Biochem Biophys Res Commun. 2008 367:768-74.

36. Labreuche Y, Veloso A, de la Vega E, Gross PS, Chapman RW, Browdy CL, et al. Nonspecific activation of antiviral immunity and induction of RNA interference may engage the same pathway in the Pacific white leg shrimp Litopenaeus vannamei. Dev Comp Immunol. $201034: 1209-18$.

37. Förstemann K, Horwich M, Wee L, Tomari Y, Zamore P. Drosophila microRNAs are sorted into functionally distinct argonaute complexes after production by dicer-1. Cell. 2007 130:287-97.

38. Scadden ADJ. The RISC subunit Tudor-SN binds to hyper-edited double-stranded RNA and promotes its cleavage. Nat Struct Mol Biol. 2005 12:489-96.

39. Phetrungnapha A, Panyim S, Ongvarrasopone C. A Tudor staphylococcal nuclease from Penaeus monodon: cDNA cloning and its involvement in RNA interference. Fish Shellfish Immunol. 2011 31:373-80.

40. Sadler AJ, Williams BR. Interferon-inducible antiviral effectors. Nat Rev Immunol. 2008 8:559-68.

41. Sabin LR, Hanna SL, Cherry S. Innate antiviral immunity in Drosophila. Curr Opin Immunol. 2010 22:4-9.

42. Kemp C, Imler JL. Antiviral immunity in drosophila. Curr Opin Immunol. 2009 21:3-9.

43. Xie Z, Qi X. Diverse small RNA-directed silencing pathways in plants. Biochim Biophys Acta. 2008 1779:720-4.

44. Aliyari R, Ding S-W. RNA-based viral immunity initiated by the Dicer family of host immune receptors. Immunol Rev. 2009 227:176-88.

45. Zhang $Y$, Song L, Zhao J, Wang L, Kong $P$, Liu L, et al. Protective immunity induced by CpG ODNs against white spot syndrome virus (WSSV) via intermediation of virus replication indirectly in Litopenaeus vannamei. Dev Comp Immunol. 2010 34:418-24.

46. Flegel T, Sritunyalucksana K. Shrimp Molecular Responses to Viral Pathogens. Mar Biotechnol (NY). 2010 13:587-607.

47. Bushati N, Cohen SM. microRNA functions. Annu Rev Cell Dev Biol. 2007 23:175-205.

48. Siomi H, Siomi MC. Posttranscriptional regulation of microRNA biogenesis in animals. Mol Cell. 2010 38:323-32. 
49. Gantier MP. New Perspectives in MicroRNA Regulation of Innate Immunity. J Interferon Cytokine Res. 2010 30:283-9.

50. Sabin LR, Zhou R, Gruber JJ, Lukinova N, Bambina S, Berman A, et al. Ars2 regulates both miRNA- and siRNA- dependent silencing and suppresses RNA virus infection in Drosophila. Cell. 2009 138:340-51.

51. Ou J, Meng Q, Li Y, Xiu Y, Du J, Gu W, et al. Identification and comparative analysis of the Eriocheir sinensis microRNA transcriptome response to Spiroplasma eriocheiris infection using a deep sequencing approach. Fish Shellfish Immunol. 2012 32:345-52.

52. Colbourne JK, Pfrender ME, Gilbert D, Thomas WK, Tucker A, Oakley TH, et al. The Ecoresponsive Genome of Daphnia pulex. Science. 2011 331:555-61.

53. Krishnan P, Babu PG, Saravanan S, Rajendran KV, Chaudhari A. DNA constructs expressing long-hairpin RNA (IhRNA) protect Penaeus monodon against White Spot Syndrome Virus. Vaccine. 2009 27:3849-55.

54. Ruan L, Bian X, Ji Y, Li M, Li F, Yan X. Isolation and identification of novel microRNAs from Marsupenaeus japonicus. Fish Shellfish Immunol. 2011 31:334-40.

55. Chen YH, Zhao L, Jia XT, Li XY, Li CZ, Yan H, et al. Isolation and characterization of cDNAs encoding Ars2 and Pasha homologues, two components of the RNA interference pathway in Litopenaeus vannamei. Fish Shellfish Immunol. 2012 32:373-80.

56. Tomari Y, Du T, Zamore PD. Sorting of Drosophila small silencing RNAs. Cell. 2007 130:299-308.

57. Fullaondo A, Lee SY. Identification of putative miRNA involved in Drosophila melanogaster immune response. Dev Comp Immunol. 2012 36:267-73.

58. Lu L-F, Liston A. MicroRNA in the immune system, microRNA as an immune system. Immunology. 2009 127:291-8.

59. Tian F, Luo J, Zhang $\mathrm{H}$, Chang $\mathrm{S}$, Song J. MiRNA expression signatures induced by Marek's disease virus infection in chickens. Genomics. 2011 in Press.

60. Li Y, Li J, Belisle S, Baskin CR, Tumpey TM, Katze MG. Differential microRNA expression and virulence of avian, 1918 reassortant, and reconstructed 1918 influenza A viruses. Virology. 2011 421:105-13.

61. Du P, Wu J, Zhang J, Zhao S, Zheng H, Gao G, et al. Viral Infection Induces Expression of Novel Phased MicroRNAs from Conserved Cellular MicroRNA Precursors. PLoS Pathog. 2011 7:e1002176.

62. Davey NE, Travé G, Gibson TJ. How viruses hijack cell regulation. Trends Biochem Sci. 2011 36:159-69.

63. Saito T, Gale Jr M. Principles of intracellular viral recognition. Curr Opin Immunol. 2007 19:17-23.

64. Loo YM, Gale MJ. Immune signaling by RIG-I-like receptors. Immunity. 2011 34:680-92.

65. O'Neill LA, Bowie AG. Sensing and signaling in antiviral innate immunity. Curr Biol. 2010 20:R328-33. 
66. Robalino J, Bartlett TC, Chapman RW, Gross PS, Browdy CL, Warr GW. Doublestranded RNA and antiviral immunity in marine shrimp: inducible host mechanisms and evidence for the evolution of viral counter-responses. Dev Comp Immunol. 2007 31:539-47.

67. Shekhar M, Lu Y. Application of Nucleic-acid-based Therapeutics for Viral Infections in Shrimp Aquaculture. Mar Biotechnol (NY). 2009 11:1-9.

68. He N, Qin Q, Xu X. Differential profile of genes expressed in hemocytes of white spot syndrome virus-resistant shrimp (Penaeus japonicus) by combining suppression subtractive hybridization and differential hybridization. Antiviral Res. 2005 66:39-45.

69. Kongton K, Phongdara A, Tonganunt-Srithaworn M, Wanna W. Molecular cloning and expression analysis of the interferon- $\gamma$-inducible lysosomal thiol reductase gene from the shrimp Penaeus monodon. Mol Biol Rep. 2011 38:3463-70.

70. Johnson $\mathrm{KN}$, van Hulten MCW, Barnes AC. "Vaccination" of shrimp against viral pathogens: Phenomenology and underlying mechanisms. Vaccine. 2008 26:4885-92.

71. Pope EC, Powell A, Roberts EC, Shields RJ, Wardle R, Rowley AF. Enhanced Cellular Immunity in Shrimp Litopenaeus vannamei after 'Vaccination'. PLoS One. 2011 6:e20960.

72. Elbashir SM, Harborth J, Lendeckel W, Yalcin A, Weber K, Tuschl T. Duplexes of 21nucleotide RNAs mediate RNA interference in cultured mammalian cells. Nature. 2001 411:494-8.

73. Marques JT, Williams BRG. Activation of the mammalian immune system by siRNAs. Nat Biotech. 2005 23:1399-405.

74. Westenberg $M$, Heinhuis $B$, Zuidema D, Vlak JM. siRNA injection induces sequenceindependent protection in Penaeus monodon against white spot syndrome virus. Virus Res. 2005 114:133-9.

75. Judge A, MacLachlan I. Overcoming the innate immune response to small interfering RNA. Hum Gene Ther. 2008 19:111-24.

76. Forsbach A, Müller C, Montino C, Kritzler A, Curdt R, Benahmed A, et al. Impact of delivery systems on siRNA immune activation and RNA interference. Immunol Lett 2012 141:169-80.

77. Saitoh S-I, Miyake K. Regulatory molecules required for nucleotide-sensing Toll-like receptors. Immunol Rev. 2009 227:32-43.

78. Takeuchi O, Akira S. Innate immunity to virus infection. Immunol Rev. 2009 227:75-86.

79. Yang L-S, Yin Z-X, Liao J-X, Huang X-D, Guo C-J, Weng S-P, et al. A Toll receptor in shrimp. Mol Immunol. 2007 44:1999-2008.

80. Wang P-H, Liang J-P, Gu Z-H, Wan D-H, Weng S-P, Yu X-Q, et al. Molecular cloning, characterization and expression analysis of two novel Tolls (LvToll2 and LvToll3) and three putative Spätzle-like Toll ligands (LvSpz1-3) from Litopenaeus vannamei. Dev Comp Immunol. 2012 36:359-71.

81. Yang C, Zhang J, Li F, Ma H, Zhang Q, Jose Priya TA, et al. A Toll receptor from Chinese shrimp Fenneropenaeus chinensis is responsive to Vibrio anguillarum infection. Fish Shellfish Immunol. 2008 24:564-74. 
82. Arts JA, Cornelissen FH, Cijsouw T, Hermsen T, Savelkoul HF, Stet RJ. Molecular cloning and expression of a Toll receptor in the giant tiger shrimp, Penaeus monodon. Fish Shellfish Immunol. 2007 23:504-13.

83. Mekata T, Kono T, Yoshida T, Sakai M, Itami T. Identification of cDNA encoding Toll receptor, MjToll gene from kuruma shrimp, Marsupenaeus japonicus. Fish Shellfish Immunol. 2008 24:122-33.

84. Labreuche $\mathrm{Y}$, O'Leary N, de la Vega E, Veloso A, Gross P, Chapman RW, et al. Lack of evidence for Litopenaeus vannamei Toll receptor (IToll) involvement in activation of sequence-independent antiviral immunity in shrimp. Dev Comp Immunol. 2009 33:806-10.

85. Gantier MP, Williams BR. The response of mammalian cells to double-stranded RNA. Cytokine Growth Factor Rev. 2007 18:363-71.

86. Nallagatla SR, Toroney R, Bevilacqua PC. Regulation of innate immunity through RNA structure and the protein kinase PKR. Curr Opin Struct Biol. 2011 21:119-27.

87. Clerzius G, Gélinas J-F, Gatignol A. Multiple levels of PKR inhibition during HIV-1 replication. Rev Med Virol 2011 21:42-53.

88. Patel CV, Handy I, Goldsmith T, Patel RC. PACT, a stress-modulated cellular activator of interferon-induced double-stranded RNA-activated protein kinase, PKR J Biol Chem. 2000 275:37993- 8.

89. Sanghvi VR, Steel LF. The Cellular TAR RNA Binding Protein, TRBP, Promotes HIV-1 Replication Primarily by Inhibiting the Activation of Double-Stranded RNA-Dependent Kinase PKR. J Virol. 2011 85:12614-21.

90. Pitaluga AN, Mason PW, Traub-Cseko YM. Non-specific antiviral response detected in RNA-treated cultured cells of the sandfly, Lutzomyia longipalpis. Dev Comp Immunol. 2008 32:191-7.

91. Sakashita K, Tatsuke T, Lee JM, Kawaguchi Y, Kusakabe T. Sequence-Nonspecific Suppression of Gene Expression by Double-stranded RNA in Silkworm Cultured Cells. J Insect Biotech Seric. 2009 78:33-7.

92. Brigneti G, Voinnet O, Li WX, Ji LH, Ding SW, Baulcombe DC. Viral pathogenicity determinants are suppressors of transgene silencing in Nicotiana benthamiana. EMBO J. 1998 17:6739-46.

93. Bivalkar-Mehla S, Vakharia J, Mehla R, Abreha M, Kanwar JR, Tikoo A, et al. Viral RNA silencing suppressors (RSS): Novel strategy of viruses to ablate the host RNA interference (RNAi) defense system. Virus Res. 2011 155:1-9.

94. Ding SW, Voinnet O. Antiviral immunity directed by small RNAs. Cell. 2007 130:413-26.

95. Li F, Ding SW. Virus counterdefense: diverse strategies for evading the RNA-silencing immunity. Annu Rev Microbiol. 2006 60:503-31.

96. Gross PS, Bartlett TC, Browdy CL, Chapman RW, Warr GW. Immune gene discovery by expressed sequence tag analysis of hemocytes and hepatopancreas in the Pacific White Shrimp, Litopenaeus vannamei, and the Atlantic White Shrimp, L. setiferus. Dev Comp Immunol. 2001 25:565-77. 
97. Cowley JA, McCulloch RJ, Spann KM, Cadogan LC, Walker PJ. Preliminary molecular and biological characterization of Mourilyan virus (MoV): a new Bunya-related virus of penaeid prawns. Diseases in Asian Aquaculture V: Proceedings of the Fifth Symposium on diseases in Asian Aquaculture. 2005 Walker PJ, Lester RG, Bondad-Reantaso MG. (eds).

98. Oanh DT, van Hulten MC, Cowley JA, Walker PJ. Pathogenicity of gill-associated virus and Mourilyan virus during mixed infections of black tiger shrimp (Penaeus monodon). J Gen Virol. 2011 92:893-901.

99. Jin $\mathrm{H}$, Zhu J-K. A viral suppressor protein inhibits host RNA silencing by hooking up with Argonautes. Genes Dev. 2010 24:853-6.

100. Chapman RW, Robalino J, F Trent Hr. EcoGenomics: analysis of complex systems via fractal geometry. Integr Comp Biol. 2006 46:902-11.

101. Robalino J, Almeida JS, McKillen D, Colglazier J, Trent HFr, Chen YA, et al. Insights into the immune transcriptome of the shrimp Litopenaeus vannamei: tissue-specific expression profiles and transcriptomic responses to immune challenge. Physiol Genomics. 2007 29:44-56.

102. Veloso A, Warr GW, Browdy CL, Chapman RW. The transcriptomic response to viral infection of two strains of shrimp (Litopenaeus vannamei). Dev Comp Immunol. 2011 35:2416.

\section{Figure}

Figure 1. Schematic representation of the miRNA and siRNA pathways in Drosophila and comparison with a putative model for RNA interference mechanisms in penaeid shrimp.

In flies, the miRNA and siRNA pathways are substantially separated. Small RNA duplexes are produced by different Dicer enzymes (Dcr1 and Dcr2) and predominantly sorted to functionally distinct Argonautes (AGO1 and AGO2) [37], although recent studies have revealed some level of cross talk between these pathways [28]. In shrimp, it remains to be determined whether LvDcr1 and LvDcr2 possess such specialized functional activities in the processing of small effector RNA duplexes. Previous studies have established that LvAgo2 physically interacts with LvDcr2 and LvTRBP [21], suggesting that they may belong to the same molecular pathway. Because knockdown of shrimp TRBP impairs dsRNA-mediated silencing of other target mRNAs [32], and considering the fact that Lvago2 expression only responds to dsRNAs [36], we propose in this model that these proteins may be required for gene silencing and the generation of siRNAs from exogenous dsRNAs.

In flies, endogenous primary-miRNA (pri-miRNA) transcripts are processed into precursor miRNAs (pre-miRNAs) in the nucleus by a protein complex formed by Drosha and its binding partners (Pasha and Ars2). The pre-miRNAs are subsequently exported in the cytoplasm and cleaved by Dcr1 into mature miRNAs, which are finally incorporated into an AGO1dependent RISC to mediate translational repression of target transcripts. Homologues of proteins involved in miRNA biogenesis (LvArs2 and LvPasha) have been identified in shrimp [55], and several miRNAs characterized [54]. Moreover, LvArs2 have been shown to interact with LvPasha and LvDcr2 [55], suggesting that this protein may also function in the production of miRNAs, similar to the role played by its homologue in flies. However, it remains to be formally demonstrated whether a miRNA pathway may also be operating in this organism. 

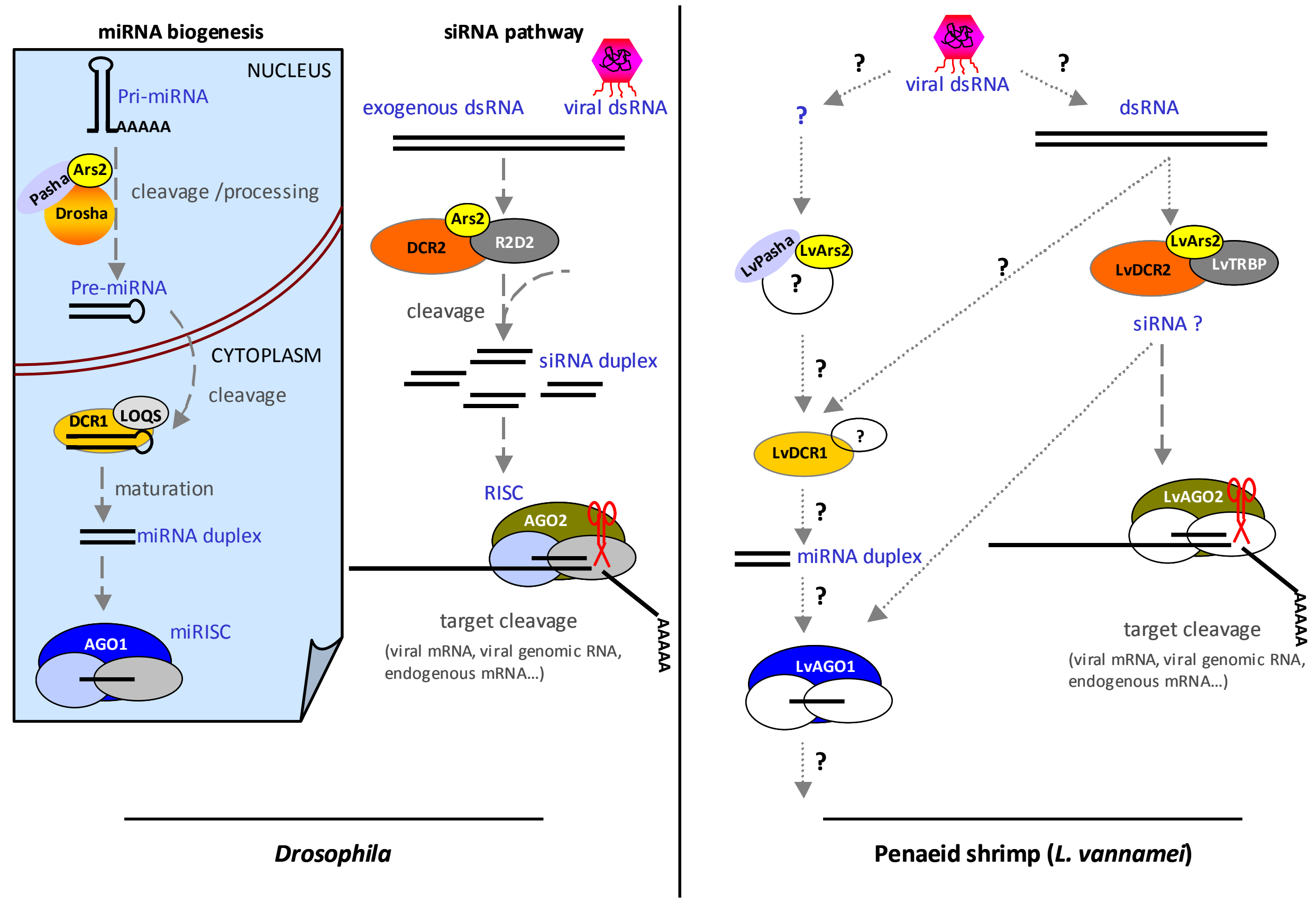

Figure 1 


\section{Table}

Table 1. Key proteins implicated in RNAi pathways of various organisms and known homologues in shrimp (modified from [16]). Nd: no avalaible data. Abbreviations: AGO, Argonaute; ALG, Argonaute-like; AUB, Aubergine; DCL, Dicer-like; DCR, Dicer; DGCR8, DiGeorge Critical Region 8, DRSH, drosha; ERGO, Endogenous RNAi deficient Argonaute; HYL-1, hyponastic leaves-1; LOQS, loquacious; PACT, protein activator of PKR; PASH, pasha; PRG, piwi-related gene; RDE-1, RNAi-deficient-4; TRBP, transactivating response (TAR) RNA-binding protein.

\begin{tabular}{|c|c|c|c|c|c|c|c|c|c|}
\hline \multirow{2}{*}{ protein } & \multirow{2}{*}{$\begin{array}{c}\text { mode of } \\
\text { action }\end{array}$} & \multirow{2}{*}{ Plant } & \multirow{2}{*}{ Nematode } & \multirow{2}{*}{ Fruitfly } & \multirow{2}{*}{ Human } & \multicolumn{4}{|c|}{ Penaeid shrimp } \\
\hline & & & & & & P. monodon & L. vannamei & M. japonicus & F. chinensis \\
\hline $\begin{array}{c}\text { Argonautes } \\
\text { AGO } \\
\text { subfamily }\end{array}$ & $\begin{array}{c}\text { components of } \\
\text { RISC }\end{array}$ & $\begin{array}{l}\text { AGO 1-7 } \\
\text { AGO10 } \\
3 \text { others }\end{array}$ & $\begin{array}{l}\text { ALG-1 } \\
\text { ALG-2 } \\
3 \text { others }\end{array}$ & $\begin{array}{l}\text { AGO1 } \\
\text { AGO2 }\end{array}$ & AGO 1- 4 & $\begin{array}{l}\text { Pm-Ago / } \\
\text { PemAGO } \\
\text { (isoform) }\end{array}$ & $\begin{array}{l}\text { Lv-Ago1 } \\
\text { Lv-Ago2 }\end{array}$ & MjAgo & nd \\
\hline $\begin{array}{c}\text { PIWI } \\
\text { subfamily }\end{array}$ & $\begin{array}{c}\text { germ cell } \\
\text { development }\end{array}$ & None & $\begin{array}{c}\text { ERGO-1 } \\
\text { PRG-1 } \\
\text { PRG-2 }\end{array}$ & $\begin{array}{l}\text { AGO3 } \\
\text { PIWI } \\
\text { AUB }\end{array}$ & $\begin{array}{c}\text { HILI } \\
\text { HIWI } \\
\text { HIWI2 } \\
\text { PIWIL3 }\end{array}$ & nd & nd & nd & nd \\
\hline $\begin{array}{c}\text { dsRNA- } \\
\text { binding } \\
\text { domain- } \\
\text { containing } \\
\text { cofactor of } \\
\text { Rnase III }\end{array}$ & $\begin{array}{l}\text { stabilizing } \\
\text { Dicer }\end{array}$ & HYL1 & $\begin{array}{c}\text { PASH-1 } \\
\text { RDE-4 }\end{array}$ & $\begin{array}{l}\text { PASHA } \\
\text { R2D2 } \\
\text { LOQS }\end{array}$ & $\begin{array}{c}\text { DGCR8 } \\
\text { TRBP } \\
\text { PACT }\end{array}$ & nd & $\begin{array}{l}\text { LvTRBP } \\
\text { LvPasha }\end{array}$ & $\mathrm{Mj}$-TRBP & Fc-TRBP \\
\hline $\begin{array}{c}\text { Arsenite } \\
\text { resistance gene } \\
\text { (Ars2) }\end{array}$ & $\begin{array}{l}\text { enhancing } \\
\text { Drosha } \\
\text { processing }\end{array}$ & SERRATE & E01A2.2 & DmArs2 & Ars2 & nd & LvArs2 & nd & nd \\
\hline
\end{tabular}

